\title{
Persistent Sciatalgia due to a Wandering Bipolar Forceps Tip after Posterior Lumbar Stabilization: A Case Report
}

\author{
Cengiz Gomleksiz ${ }^{1}$ Mehmet Tokmak ${ }^{1} \quad$ Umut Yaka $^{1}$ Erhan Arslan ${ }^{2}$ Muhammed Arif Özbek ${ }^{1}$ \\ S. Baki Albayrak ${ }^{1}$ \\ ${ }^{1}$ Department of Neurosurgery, Istanbul Medipol University, i̇stanbul, \\ Turkey \\ ${ }^{2}$ Department of Neurosurgery, Mengücek Gazi Training and Research \\ Hospital, Erzincan University, Erzincan, Turkey \\ Address for correspondence S. Baki Albayrak, MD, Department of \\ Neurosurgery, Istanbul Medipol University, Atatürk Bulvarı No:27, \\ 34083 Unkapanı/Fatih/istanbul, Turkey \\ (e-mail: serdarbaki@gmail.com).
}

Indian J Neurosurg 2016;5:34-36.

\begin{abstract}
There are several reports in the literature about the retained surgical materials as a cause of radicular pain or sensory impairment after spinal surgery. We report a patient

Keywords

- sciatalgia

- wandering

- bipolar with a persistent radicular pain in the distribution of left S1 nerve dermatome following lumbar stabilization surgery. Retained bipolar forceps tip has not been reported previously in the literature as a cause of sciatalgia after posterior lumbar surgery.
\end{abstract}

\section{Introduction}

Persistent radicular pain after posterior lumbar stabilization occurs and it mostly results from root irritation by a pedicle screw or a residual disc herniation. However, radicular pain after spinal surgeries occurs very rarely due to retained surgical materials such as sponges, cottonoids, etc. There are only few cases in the literature that the forgotten surgical instruments in surgical sites were the source of the persistent radicular pain. ${ }^{1-4}$

\section{Case Report}

A 44-year-old woman with a history of posterior lumbar stabilization surgery 2 years before was referred to the neurosurgery outpatient clinic with severe left leg pain over the left S1 dermatome. Left S1 hypoesthesia and straight leg raising positivity were detected. There was no muscle weakness or sphincter function impairment. Blood tests did not reveal any evidence of systemic inflammation. Lumbosacral plain radiographs demonstrated an opaque material on the left side of the L5-S1 disc space (-Fig. 1A, B). We ordered a lumbosacral CT and a hyperdense area was clearly delineated in the left S1 lateral recess (- Fig. 1C), after we noted a metallic artifact effect in the lumbosacral MR images (-Fig. 1D).

Because nonsteroidal anti-inflammatory medication for the treatment of sciatalgia was ineffective, surgical exploration and removal of the potential retained material was planned. Left L5-S1 fenestration and left S1 foraminotomy were performed under general anesthesia. Under surgical microscope, we observed that the left S1 nerve root was swollen and hyperemic. Upon retracting the nerve root medially, a retained metallic material abutted into the vertebral body at the lateral recess was exposed. Bony tissue of the vertebral body around the metallic material was drilled with a high-speed electric drill and the metallic foreign material was removed. Immediately after surgery, left leg pain resolved completely with full muscle strength. We thought that the removed material was likely an end piece of a shattered bipolar forceps (-Fig. 1E). Post-op radiographs confirmed the removal of the foreign metallic material (-Fig. 1F).
License terms of India received

June 1, 2015

accepted

November 18, 2015

published online

February 19, 2016
DOI http://dx.doi.org/

10.1055/s-0036-1572372.

ISSN 2277-954X. 


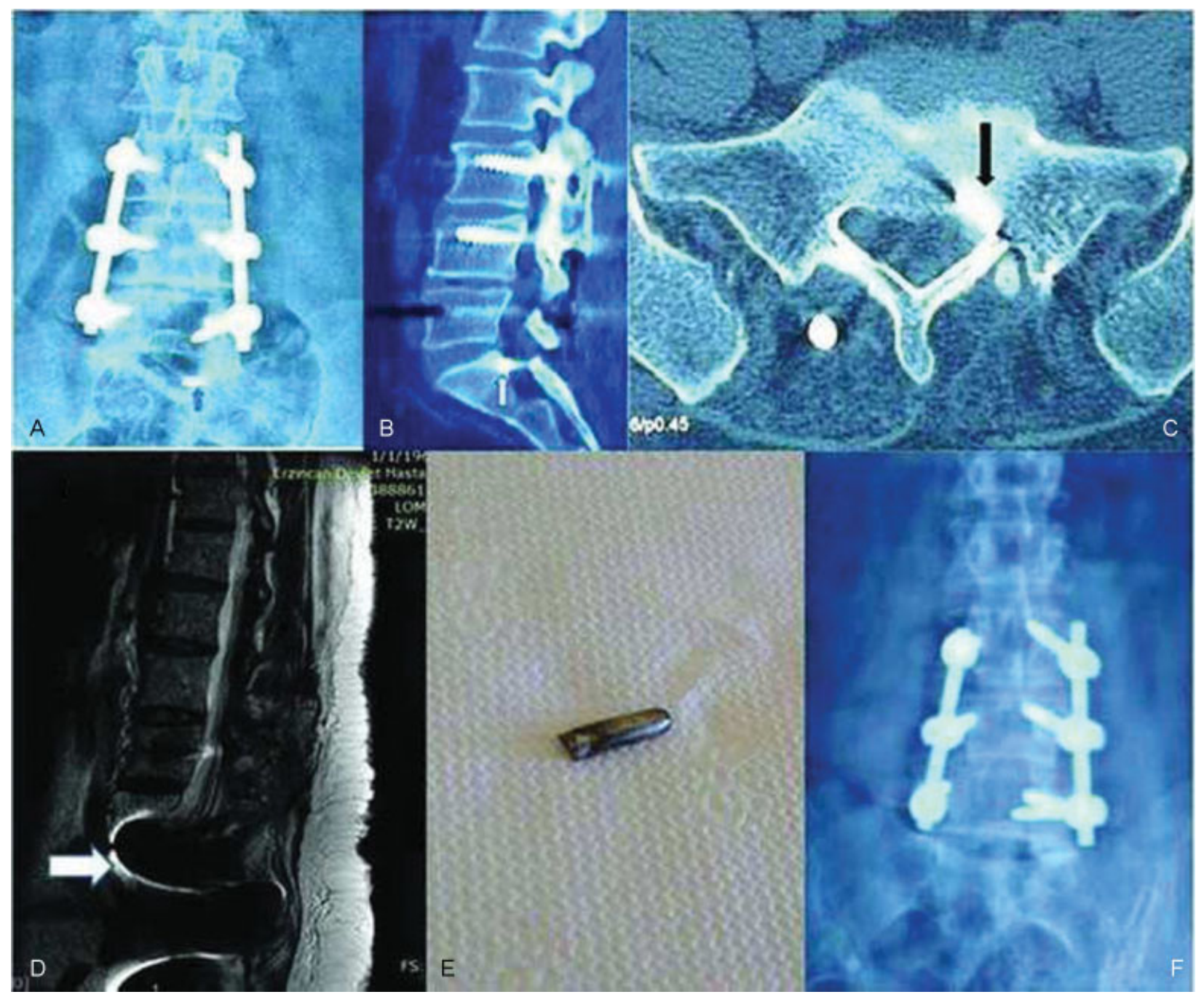

Fig. 1 (A, B) Preoperative A-P and lateral plain lumbosacral radiographies showing an opaque material at the left side of the L5-S1 disc space (black and white arrows, respectively). (C) Lumbosacral CT scan showing the hyperdense material at the left S1 lateral recess (black arrow). (D) Lumbosacral T2W sagittal MRI showing metallic artifact at the L5-S1 level (white arrow). (E) Photograph of the removed bipolar forceps tip. (F) Postoperative plain lumbosacral radiography showing removal of the bipolar forceps tip.

\section{Discussion}

Although there are various reports regarding the retained surgical materials such as epidural catheter tip, surgical gauze, etc., retained bipolar forceps tip as a cause of radiculopathy has not been reported so far. ${ }^{3,5,6}$ Surgical instruments are prone to fatigue in the same way as other metallic materials and they can be broken during surgery. In our case, the most likely scenario was that a bipolar forceps tip was broken and dropped into the surgery site, and the surgeon was not aware of this incident. After surgery, the patient suffered from episodic left leg pain, and these episodes gradually became more frequent and resistant to medical treatment. Interestingly, the superior border of the surgery site was the L3 level, However, the bipolar tip appeared at the left S1 lateral recess in radiographs. No laminectomy defect at the L5 and S1 vertebra was observed intraoperatively. Thus, this fact was explained by the gravitational force causing downward migration of the bipolar tip. While examining magnetic resonance (MR) images at first sight, a broken piece of rod was suspected, but the metallic artifact in magnetic resonance imaging (MRI) refuted this hypothesis as the rods used in contemporary neurosurgery are made of titanium that does not produce any metallic artifacts in MRI. We therefore concluded that the metallic material might be a piece of broken surgical hand tool. Further imaging techniques including computed tomography (CT) and MRI should be performed to confirm the diagnosis and to provide a more clear demonstration of the site of the retained material and the surrounding structures. In this case, the patient was fortunate as bipolar tip with a relatively pointed and sharp tip did not cause any dural injuries and infection with a potentially devastating outcome.

\section{Conclusion}

Bipolar forceps are perhaps the most important and indispensable surgical instrument for neurosurgeons in modern neurosurgery practice. If we draw an analogy of an 
36 Persistent Sciatalgia due to a Wandering Bipolar Gomleksiz et al.

operation to a battlefield, bipolars are the cannons of the neurosurgeons. However, this case report reminds us once again that we should not rely on the integrity and durability of any instruments, including bipolar forceps canons of neurosurgeons.

\section{References}

1 Cherian RA, Haq N. Case report: Acute paraplegia due to surgical related thoracic cord compression. Indian J Radiol Imaging 1999; $9(2): 49-51$

2 Mulder M, Crosier J, Dunn R. Cauda equina compression by hydrogel dural sealant after a laminotomy and discectomy: case report. Spine 2009;34(4):E144-E148
3 Partheni M, Kalogheropoulou C, Karageorgos N, Panagiotopoulos V, Voulgaris S, Tzortzidis F. Radiculopathy after lumbar discectomy due to intraspinal retained Surgicel: clinical and magnetic resonance imaging evaluation. Spine J 2006;6(4): 455-458

4 Wada E, Yonenobu K, Ebara S, Kuwahara O, Ono K. Epidural migration of hemostatic agents as a cause of postthoracotomy paraplegia. Report of two cases. J Neurosurg 1993;78(4): 658-660

5 Rajković Z, Altarac S, Papeš D. An unusual cause of chronic lumbar back pain: retained surgical gauze discovered after 40 years. Pain Med 2010;11(12):1777-1779

6 Staats PS, Stinson MS, Lee RR. Lumbar stenosis complicating retained epidural catheter tip. Anesthesiology 1995;83(5): $1115-1118$ 